\title{
Scale Invariance and similar Invariance Conditions for Bankruptcy Problems
}

\author{
Thierry Marchant \\ Ghent University \\ Thierry. Marchant@UGent . be
}

March 2008

\begin{abstract}
A frequent motivation for the use of Scale Invariance in the bankruptcy literature is that it imposes that the outcome of a bankruptcy problem does not depend on the units of measurement. We show that this interpretation is not correct. Scale Invariance is an invariance condition that applies when all amounts are multiplied by a constant (without change of units). With this interpretation in mind, it is natural to consider other invariance conditions, for example one that applies when all amounts are increased by the same constant. In this paper, we analyze the consequences of several invariance conditions.
\end{abstract}

\section{Introduction}

A bankruptcy problem occurs when one has to divide a non-negative amount $E \in \mathbb{R}_{+}$of a resource between several agents (the population of agents is finite and denoted by $N \subseteq \mathbb{N}$ ), each having his own non-negative claim or demand $c_{i}$, with the constraint that $\sum c_{i} \geq E$ (a summation without explicit bounds applies over all agents in $N)$. The vector of claims is denoted by $c=\left(c_{1}, \ldots, c_{n}\right), n$ being the size of the population. Let $\mathscr{C}^{N}$ be the class of all bankruptcy problems for the population $N$. We will allow the population $N$ to vary. Let $\mathscr{N}$ be the set of all non-empty finite subsets of $\mathbb{N}$. We denote by $\mathscr{C}$ the class of all bankruptcy problems for all populations $N$, i.e., $\mathscr{C}=\bigcup_{N \in \mathscr{N}} \mathscr{C}^{N}$.

A division rule is a mapping that assigns to each agent in $N$ his share of the amount $E$. Formally, it is a mapping $R: \mathscr{C} \mapsto \bigcup_{N \in \mathscr{N}} \mathbb{R}_{+}^{N}:(c, E) \rightarrow R(c, E)$ satisfying, for all $N \in \mathscr{N}$ and all $(c, E) \in \mathscr{C}^{N}$,

- $R(c, E) \in \mathbb{R}_{+}^{N}$ and

- $0 \leq R_{i}(c, E) \leq c_{i}$ for all $i$ in $N$.

According to this definition, $R_{i}(c, E)$ is the share of agent $i$. 
It is often required that a division rule satisfies some conditions supposed to make it a fair, rational or consistent rule. For example, continuity, resource monotonicity (i.e., monotonicity w.r.t. $E$ ), symmetry w.r.t. $c_{i}$, and so on. Another condition is Scale Invariance. It has been introduced in the bankruptcy literature by Young [1988] and used later on by Moulin [2000, 2002], among others. It is also used in very similar problems by other authors, e.g., in cost sharing by Friedman [2004]. It can be formulated as follows.

A 1 Scale Invariance. For all $(c, E) \in \mathscr{C}$,

$$
R(\gamma c, \gamma E)=\gamma R(c, E), \quad \forall \gamma>1^{1} .
$$

The justification given by [Young, 1988, p.323] for this condition is

[... the relative distribution of taxes is assumed to be independent of the units in which taxable income is measured [...]

Moulin [2002] writes

Scale invariance $[\ldots]$ is an impeccable axiom insisting that the choice of the unit to measure both the demands/claims/taxable income and the available resources, should be of no consequence whatsoever.

Similar scale invariance conditions can be found in different parts of the economic literature and, often, the same motivation is given: everything should be independent of the units of measurement ${ }^{2}$. Nevertheless, in the context of bargaining theory, [Roemer, 1996, Section 2.5] has shown that Scale Invariance is not about changes of units but about the way small and large amounts are treated.

In the bankruptcy context, the 'change of units' interpretation is also wrong, as we now show. First, it is important to remark that Scale Invariance puts strong constraints on division rules even if no change of units of measurement is considered. Indeed, suppose $N=\{1,2\}$ and $R((10,20), 15)=(5,15)$ where all numbers represent amounts in Euros. Suppose also that, using the same division rule $R$, we want to share 150 Euros among the same agents (perhaps at a different moment) with claims 100 and 200 Euros. Then, by scale Invariance, we necessarily obtain $R((100,200), 150)=(50,100)$ where all numbers still represent amounts in Euros and, more generally, $R((\gamma 10, \gamma 20), \gamma 15)=(\gamma 5, \gamma 10)$. This is just a homogeneity condition, saying that large amounts are treated in the same way as small ones, and does not imply any change of unit.

Remark now that $R$ is defined as a mapping from $\mathscr{C}$ into $\bigcup_{N \in \mathscr{N}} \mathbb{R}_{+}^{N}$. No reference is made in the definition to some units. So, formally speaking, a division rule does not share Pounds, Yens or Dirhams but only real numbers.

\footnotetext{
${ }^{1}$ This condition is often imposed for all $\gamma>0$ but it is easy to see that both conditions are equivalent

${ }^{2}$ Note that Thomson [2003] uses the name Homogeneity for this condition and does not motivate it by changes of unit of measurement.
} 
We may of course interpret elements of $\mathbb{R}_{+}$as amounts in Euros (or Rupees, Leones, ...). But if we do so, then $R$ should be considered as a division rule for Euros and only Euros (resp. Rupees, Leones, ...). We cannot change. If we consider changes of units with a notation that does not take units into account, there are chances that our reasoning be sloppy. For instance, if ' 1 ' represents one dollar, then '100' can represent hundred dollars but also hundred cents. Hence, any number can represent anything.

So, Scale Invariance is not really a scale invariance condition ${ }^{3}$ in the sense of invariance w.r.t. changes of units. This does not mean that Scale Invariance is not interesting. Invariance conditions of all kinds have proven useful in many domains. They can express, among others, empirically observable relations or normative principles and can help understand many models. They are very powerful tools for the researcher trying to axiomatically characterize a model, a procedure or some structure. When we have a set of ethically appealing axioms and this set characterizes a family of division rules, we can further impose an invariance condition in order to isolate an ethically grounded rule that is also mathematically convenient. That is why, in the rest of this paper, we will consider and analyze the consequences of Scale Invariance and several similar invariance conditions. We begin with two conditions expressing an invariance with respect to the multiplication by a constant (Section 2). We will then consider invariance conditions applying when resources and/or claims are modified by the addition of a constant and no longer by a multiplication (Section 3). Instead of considering each conceivable invariance condition in turn, we will try in Section 4 to consider a large family of invariance conditions. Finally, in Section 5, we will introduce a last invariance condition that, unlike scale invariance, is compatible with an ethical principle introduced by Young [1987].

\section{Invariance with respect to the multiplication by a constant}

We consider two conditions expressing an invariance with respect to the multiplication by a constant. The first one is Scale Invariance. The next one will be called Multiplicative Invariance.

\subsection{Scale Invariance}

In the rationing literature, Scale Invariance has always been considered in conjunction with other conditions. In this section, we look at the consequences of Scale Invariance alone.

Let $R$ be any division rule. For any $N$ and any $(c, E) \in \mathscr{C}^{N}$, define then the

\footnotetext{
${ }^{3}$ If we use the word 'scale' as in 'large scale', 'small scale' or 'scale economies', then the expression 'scale invariance' is right. Not if we use it as in 'measurement scale,' as is common in the literature.
} 
mapping $S: \mathscr{C} \mapsto \bigcup_{N \in \mathscr{N}} \mathbb{R}_{+}^{N}$ by means of

$$
S(c, E)= \begin{cases}(0, \ldots, 0) & \text { if } c_{i}=0 \forall i \in N, \\ c_{j} R\left(\frac{c}{c_{j}}, \frac{E}{c_{j}}\right) & \text { otherwise. }\end{cases}
$$

where $c_{j}$ is any of the positive claims in $c$. It is easy to check that, for all $E: 0 \leq E \leq \sum c_{i}$, we have $0 \leq S_{i}(c, E) \leq c_{i}$. So, $S$ is a division rule. In addition, $S$ is Scale Invariant. Indeed,

$$
S(\gamma c, \gamma E)=\gamma c_{j} R\left(\frac{c}{c_{j}}, \frac{E}{c_{j}}\right)=\gamma S(c, E) .
$$

This shows that we can easily construct a Scale Invariant rule starting from any rule and, hence, that Scale Invariance is a very weak condition. Without adding some other strong conditions, we cannot say much about Scale Invariance. So, we proceed to another condition.

\subsection{Multiplicative Invariance}

Scale Invariance is nothing but an invariance with respect to the multiplication by a constant but it is not the only sensible one. Here is another one.

A 2 Multiplicative Invariance. For all $N \in \mathscr{N}$, all $(c, E) \in \mathscr{C}^{N}$ and all $i \in N$,

$$
R_{i}(c / \gamma, E)=R_{i}(c, E), \quad \forall \gamma>1 .
$$

It expresses the fact that the claims themselves are not important but that the ratios of the claims matter since ratios of claims are not affected by the division by $\gamma$. This condition is obviously equivalent to

$$
R_{i}(\gamma c, E)=R_{i}(c, E), \quad \forall \gamma: \sum \gamma c_{i} \geq E .
$$

Note that this condition applies in particular for $\gamma^{\prime}=E / C$ where $C=\sum_{j \in N} c_{j}$. Because, $\sum \gamma^{\prime} c_{i}=E$, we have

$$
R_{i}\left(\gamma^{\prime} c, E\right)=\gamma^{\prime} c_{i}=c_{i} E / C
$$

and, so, we have proved the following very simple result.

Proposition 1 A division rule satisfies Multiplicative Invariance if and only if it is the proportional rule, defined by (1).

\section{Invariance with respect to the addition of a constant}

The conditions referred to by Scale Invariance and Multiplicative Invariance are, as shown earlier, invariances with respect to a multiplication. It is therefore natural to also consider corresponding additive invariance conditions. 


\subsection{Additive Invariance 1}

Given a real number $\gamma$, let $\bar{\gamma}$ denote the vector $(\gamma, \ldots, \gamma)$ in $\mathbb{R}^{N}$.

A 3 Additive Invariance 1. For all $N \in \mathscr{N}$, all $(c, E) \in \mathscr{C}^{N}$ and all $i \in N$,

$$
R_{i}(\bar{\gamma}+c, \gamma+E)=\phi(\gamma)+R_{i}(c, E), \quad \forall \gamma>0
$$

This condition, like Scale Invariance, expresses the fact that small amounts should be treated like large amounts. But here, the transformation of a small amount into a large one is the result of an addition instead of a multiplication. Contrary to Scale Invariance, Additive Invariance 1 is a strong condition as shown in the following result.

Proposition 2 For $N=\{1,2\}$, a division rule $R$ satisfies Additive Invariance 1 if and only if it is the Contested Garment rule, defined by

$$
R_{i}(c, E)= \begin{cases}\min \left\{c_{i} / 2, z\right\} & \text { if }\left(c_{1}+c_{2}\right) / 2 \geq E, \\ c_{i}-\min \left\{c_{i} / 2, z^{\prime}\right\} & \text { if }\left(c_{1}+c_{2}\right) / 2 \leq E\end{cases}
$$

where $z$ is the solution of $\sum \min \left\{c_{i} / 2, z\right\}=E$ and $z^{\prime}$ is the solution of $c_{1}+c_{2}-$ $\sum \min \left\{c_{i} / 2, z^{\prime}\right\}=E$.

Proof. Applying several times Additive Invariance 1, we have

$$
\begin{aligned}
R_{i}\left(\bar{\gamma}+\bar{\gamma}^{\prime}+c, \gamma+\gamma^{\prime}+E\right) & =\phi(\gamma)+\phi\left(\gamma^{\prime}\right)+R_{i}(c, E) \\
& =\phi\left(\gamma+\gamma^{\prime}\right)+R_{i}(c, E),
\end{aligned}
$$

for all $\gamma, \gamma^{\prime} \geq 0$. Hence, $\phi(\gamma)+\phi\left(\gamma^{\prime}\right)=\phi\left(\gamma+\gamma^{\prime}\right)$. Because $\phi$ is clearly bounded, on any closed interval, the only solution of this Cauchy's equation is $\phi(\gamma)=k \gamma$, with $k>0$. Because the sum of the shares is always equal to the amount to be divided, we find that $k=1 / 2$. So,

$$
R_{i}(\bar{\gamma}+c, \gamma+E)=\gamma / 2+R_{i}(c, E), \forall \gamma \geq 0
$$

or

$$
R_{i}(c, E)=\gamma / 2+R_{i}(c-\bar{\gamma}, E-\gamma), \forall \gamma: 0 \leq \gamma \leq \min \left(c_{1}, c_{2}, E, c_{1}+c_{2}-E\right)
$$

Suppose $c_{2} \leq c_{1}$ and let us rewrite this condition for $\gamma=\min \left(c_{2}, E, c_{1}+c_{2}-E\right)$, we obtain

$$
\begin{aligned}
& R_{i}(c, E)=\min \left(c_{2}, E, c_{1}+c_{2}-E\right) / 2 \\
& \quad+R_{i}\left[c_{1}-\min \left(c_{2}, E, c_{1}+c_{2}-E\right), c_{2}-\min \left(c_{2}, E, c_{1}+c_{2}-E\right),\right. \\
& \left.\quad E-\min \left(c_{2}, E, c_{1}+c_{2}-E\right)\right] .
\end{aligned}
$$

We analyze now the different cases. If $0 \leq E \leq c_{2}$, we have

$$
R_{i}\left(c_{1}, c_{2}, E\right)=E / 2+R_{i}\left(c_{1}-E, c_{2}-E, 0\right)=E / 2 .
$$


Suppose now that $c_{2}<E \leq c_{1}$. We have

$$
R_{i}(c, E)=c_{2} / 2+R_{i}\left(c_{1}-c_{2}, 0, E-c_{2}\right)
$$

and, in particular, $R_{1}(c, E)=c_{2} / 2+E-c_{2}=E-c_{2} / 2$. Finally, if $c_{1}<E \leq$ $c_{1}+c_{2}$, then

$$
R_{i}(c, E)=\left(c_{1}+c_{2}-E\right) / 2+R_{i}\left(E-c_{2}, E-c_{1}, 2 E-c_{1}-c_{2}\right),
$$

and, in particular, $R_{1}(c, E)=\left(c_{1}+c_{2}-E\right) / 2+E-c_{2}=\left(E+c_{1}-c_{2}\right) / 2$. In summary, when $c_{1} \geq c_{2}$,

$$
R_{1}(c, E)= \begin{cases}E / 2 & \text { if } 0 \leq E \leq c_{2} \\ E-c_{2} / 2 & \text { if } c_{2}<E \leq c_{1} \\ \left(E+c_{1}-c_{2}\right) / 2 & \text { if } c_{1}<E \leq c_{1}+c_{2}\end{cases}
$$

A similar reasoning holds when $c_{1} \leq c_{2}$ and yields

$$
R_{1}(c, E)= \begin{cases}E / 2 & \text { if } 0 \leq E \leq c_{1} \\ c_{1} / 2 & \text { if } c_{1}<E \leq c_{2} \\ \left(E+c_{1}-c_{2}\right) / 2 & \text { if } c_{2}<E \leq c_{1}+c_{2}\end{cases}
$$

Equations (2) and (3) define a unique division rule, namely the Contested Garment rule [Aumann and Maschler, 1985].

When there are more than two agents, Additive Invariance 1 does not characterize a single rule but, if we add a very mild condition (Limited Consistency), we obtain again a characterization.

A 4 Limited Consistency. For all $N \in \mathscr{N}$ and all $(c, E) \in \mathscr{C}^{N}, c_{i}=0$ for all $i$ in $N^{\prime} \subset N$ implies $R_{j}(c, E)=R_{j}\left(c^{\prime}, E\right)$ for all $j \in N \backslash N^{\prime}$ where $c^{\prime}=\left(c_{i}\right)_{i \in N \backslash N^{\prime}}$.

This condition, first introduced by O'Neill [1982], prevents agents with a claim equal to zero to play any role. Moulin [2002] uses the same condition (named Zero Consistency) with one difference: the set $N^{\prime}$ is a singleton. Of course, if we apply several times Zero Consistency, we obtain Limited Consistency. So, these two conditions are equivalent.

Proposition $3 A$ division rule $R$ satisfies Additive Invariance 1 and Limited Consistency if and only if it is recursively defined, for all $N \in \mathscr{N}$ and all $(c, E) \in \mathscr{C}^{N}$, by

1. If $E \leq c_{(1)}$, then $R_{i}(c, E)=E / n$;

2. If $E \geq C-(n-1) c_{(1)}$, then $R_{i}(c, E)=c_{i}-\frac{C-E}{n}$;

3. If $c_{(1)} \leq E \leq C-(n-1) c_{(1)}$, then

- If $c_{i}=c_{(1)}$, then $R_{i}(c, E)=c_{(1)} / n$; 
- If $c_{i}>c_{(1)}$, then $R_{i}(c, E)=c_{(1)} / n+R_{i}\left(c_{N^{\prime}}-\bar{c}_{(1)}, E-c_{(1)}\right)$

where $c_{(1)}=\min _{j \in N} c_{j}, C=\sum_{j \in N} c_{j}, N^{\prime}=\left\{k \in N: c_{k}>c_{(1)}\right\}$ and $c_{N^{\prime}}=$ $\left(c_{k}\right)_{k \in N^{\prime}}$.

The rule characterized by this proposition is the minimal overlap rule introduced by O'Neill [1982], as pointed out to me by William Thomson. In words, this rule works as follows. When $E$ is very small (resp. very large), the gains (resp. the losses) are divided equally. For intermediate values of $E$, the agent(s) with the smallest claim receives his claim divided by the number of agents. The agent(s) with a claim larger than the smallest one receive also the smallest claim divided by the number of agents plus a bonus. This bonus is their share for a new problem solved with the same rule. In this new problem, the agent(s) with the smallest claims have been removed and the smallest claim is subtracted from the other claims and E. Fig. 1 illustrates this rule. Note that this rule belongs to the ICI family defined by Thomson [2003].

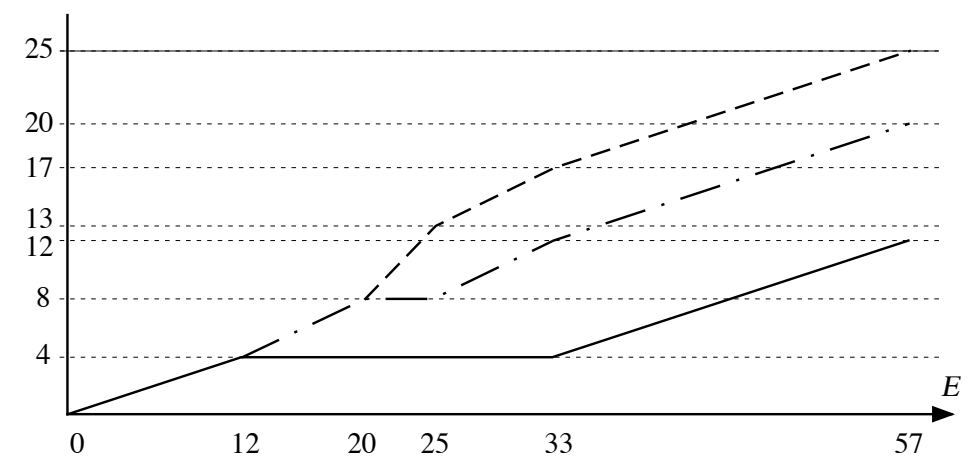

Figure 1: The rule of Prop. 3. The graph of $R_{i}(c, E)$ in function of $E$, for $c=(12,20,25)$. Solid line: $R_{1}$; dashed with dots: $R_{2}$; dashed: $R_{3}$.

Proof. Following the same reasoning as in Prop. 2, we find, for $(c, E) \in \mathscr{C}^{N}$, $\phi(\gamma)=\gamma / n$ and, hence,

$$
R_{i}(c, E)=\frac{\gamma}{n}+R_{i}(c-\bar{\gamma}, E-\gamma), \forall \gamma: 0 \leq \gamma \leq \min \left(c_{(1)}, E, \frac{C-E}{n-1}\right) .
$$

We rewrite this condition for $\gamma=\min \left(c_{(1)}, E, \frac{C-E}{n-1}\right)$ :

$$
\begin{aligned}
& R_{i}(c, E)=\frac{1}{n} \min \left(c_{(1)}, E, \frac{C-E}{n-1}\right) \\
& +R_{i}\left(c-\overline{\min \left(c_{(1)}, E, \frac{C-E}{n-1}\right)}, E-\min \left(c_{(1)}, E, \frac{C-E}{n-1}\right)\right) .
\end{aligned}
$$

We now discuss the various cases of Prop. 3. 
1. If $E \leq c_{(1)}$, we find $R_{i}(c, E)=E / n$, because $R_{i}(c, 0)=0$.

2. If $E \geq C-(n-1) c_{(1)}$, we find

$$
R_{i}(c, E)=\frac{C-E}{n(n-1)}+R_{i}\left(c-\frac{\overline{C-E}}{n-1}, E-\frac{C-E}{n-1}\right),
$$

that is $R_{i}(c, E)=c_{i}-\frac{C-E}{n}$ because, by definition of a division rule, when the amount to be divided is equal to the sum of the claims, then each agent receives exactly his claim.

3. If $c_{(1)} \leq E \leq C-(n-1) c_{(1)}$, then we have

$$
R_{i}(c, E)=c_{(1)} / n+R_{i}\left(c-\bar{c}_{(1)}, E-c_{(1)}\right) .
$$

- For the agents with $c_{i}=c_{(1)}$, we find $R_{i}(c, E)=c_{(1)} / n$ because, by definition of a rule, the share of an agent with a claim equal to zero is zero.

- For the agents with $c_{i}>c_{(1)}$, we apply Limited Consistency and we find $R_{i}(c, E)=c_{(1)} / n+R_{i}\left(c_{N^{\prime}}-\bar{c}_{(1)}, E-c_{(1)}\right)$.

The reader will easily verify that one can obtain a weighted version of this rule by imposing a weak version of Additive Invariance 1 where $\phi(\gamma)$ is replaced by $\phi_{i}(\gamma)$. The Cauchy equation in the proof then becomes a Pexider equation.

\section{$3.2 \quad$ Additive Invariance 2}

Additive Invariance 1 is in fact just one of the invariances with respect to the addition of a constant that we can think of. Here is another one.

A 5 Additive Invariance 2. For all $N \in \mathscr{N}$, all $(c, E) \in \mathscr{C}^{N}$ and all $i \in N$,

$$
R_{i}(\bar{\gamma}+c, E)=R_{i}(c, E), \quad \forall \gamma>0 .
$$

This condition, in some sense, expresses the fact that the differences between the claims matter and not the claims themselves. It is very similar to Multiplicative Invariance. Just like Multiplicative Invariance and Additive Invariance 1, this is a strong condition as shown in the following result.

Proposition 4 For $N=\{1,2\}$, a division rule $R$ satisfies Additive Invariance 2 if and only if it is the Constrained Equal Losses rule, defined by $R_{i}(c, E)=$ $\max \left(0, c_{i}-z\right)$ where $z$ is the solution of $\sum \max \left(0, c_{i}-z\right)=E$.

Proof. We can rewrite Additive Invariance 2 as

$$
R_{i}(c, E)=R_{i}(c-\bar{\gamma}, E), \forall \gamma: 0 \leq \gamma \leq \min \left(c_{(1)}, \frac{C-E}{n}\right) .
$$


Suppose $c_{2} \leq c_{1}$ and let us rewrite this condition for $\gamma=\min \left(c_{2}, \frac{C-E}{n}\right)$, we obtain

$$
R_{i}(c, E)=R_{i}\left(c_{1}-\min \left(c_{2}, \frac{C-E}{n}\right), c_{2}-\min \left(c_{2}, \frac{C-E}{n}\right), E\right) .
$$

We analyze now the different cases. If $0 \leq E \leq c_{1}-c_{2}$, we have

$$
R_{i}(c, E)=R_{i}\left(c_{1}-c_{2}, c_{2}-c_{2}, E\right),
$$

and, in particular, $R_{1}(c, E)=E$. But if $E>c_{1}-c_{2}$, then

$$
R_{i}(c, E)=R_{i}\left(c_{1}-\frac{C-E}{n}, c_{2}-\frac{C-E}{n}, E\right),
$$

and, in particular, $R_{1}(c, E)=\left(c_{1}-c_{2}\right) / 2+E / 2$. In summary, when $c_{1} \geq c_{2}$,

$$
R_{1}(c, E)= \begin{cases}E & \text { if } 0 \leq E \leq c_{1}-c_{2} \\ \left(c_{1}-c_{2}\right) / 2+E / 2 & \text { if } E>c_{1}-c_{2}\end{cases}
$$

A similar reasoning holds when $c_{1} \leq c_{2}$ and yields

$$
R_{1}(c, E)= \begin{cases}0 & \text { if } 0 \leq E \leq c_{2}-c_{1} \\ \left(c_{1}-c_{2}\right) / 2+E / 2 & \text { if } E>c_{2}-c_{1} .\end{cases}
$$

Equations (4) and (5) define a unique division rule, namely the Constrained Equal Losses rule [Aumann and Maschler, 1985].

When there are more than two agents, Additive Invariance 2 does not characterize a single rule but, once more, if we add Limited Consistency, we obtain a characterization.

Proposition 5 A division rule $R$ satisfies Additive Invariance 2 and Limited Consistency if and only if it is the Constrained Equal Losses rule, defined in Prop. 4.

Proof. As in Prop. 4, we have, for $(c, E) \in \mathscr{C}^{N}$,

$$
R_{i}(c, E)=R_{i}(c-\bar{\gamma}, E), \forall \gamma: 0 \leq \gamma \leq \min \left(c_{(1)}, \frac{C-E}{n}\right) .
$$

We rewrite this condition for $\gamma=\min \left(c_{(1)}, \frac{C-E}{n}\right)$ :

$$
R_{i}(c, E)=R_{i}\left(c-\overline{\min \left(c_{(1)}, \frac{C-E}{n}\right)}, E\right) .
$$

We now discuss two cases. 
1. If $E \geq C-n c_{(1)}$, we find

$$
R_{i}(c, E)=R_{i}\left(c-\frac{\overline{C-E}}{n}, E\right),
$$

that is $R_{i}(c, E)=c_{i}-\frac{C-E}{n}$ because, by definition of a division rule, when the amount to be divided is equal to the sum of the claims, then each agent receives exactly his claim. So, the losses are divided equally.

2. If $E<C-n c_{(1)}$, then we have

$$
R_{i}(c, E)=R_{i}\left(c-\bar{c}_{(1)}, E\right) .
$$

- For the agents with $c_{i}=c_{(1)}$, we find $R_{i}(c, E)=0$ because, by definition of a rule, the share of an agent with a claim equal to zero is zero.

- For the agents with $c_{i}>c_{(1)}$, we apply Limited Consistency and we find $R_{i}(c, E)=R_{i}\left(c_{N^{\prime}}-\bar{c}_{(1)}, E\right)$.

So, in this case, the agents with small claims receive nothing while the rest of the losses is divided, using the same rule, among the agents with a sufficiently large claim.

In summary, we find that the losses are divided equally provided that nobody receives a negative award. This is the definition of the Constrained Equal Losses rule.

\subsection{Additive Invariance 3}

Another sensible invariance with respect to the addition of a constant is

A 6 Additive Invariance 3. For all $N \in \mathscr{N}$, all $(c, E) \in \mathscr{C}^{N}$ and all $i \in N$,

$$
R_{i}(\bar{\gamma}+c, n \gamma+E)=\gamma+R_{i}(c, E), \quad \forall \gamma>0 .
$$

The reader will easily check that this condition (with Limited Consistency if $n>2$ ) characterizes a rule which is the dual of the Constrained Equal Losses rule, namely the Constrained Equal Gains rule [Aumann and Maschler, 1985]. We say that two rules $R$ and $S$ are dual of each other [Moulin, 2002] iff

$$
S(c, E)=c-R(c, C-E) .
$$

Actually, Additive Invariance 3 and Additive Invariance 2 are dual of each other, i.e., a rule satisfies one property if and only if its dual satisfies the other property. 


\section{Invariance with respect to a family of trans- formations}

Instead of writing many different invariance conditions, we now try to consider many possible invariance conditions at once. Let $\mathcal{T}=\{(\phi, \psi, \chi)\}$ such that $\phi, \psi$ and $\chi$ are strictly increasing and continuous mappings from $\mathbb{R}_{+}$into $\mathbb{R}_{+}$. So, $\mathcal{T}$ is the set of all possible triples of transformations. Let $\phi(c)$ denote the vector $\left(\phi\left(c_{1}\right), \ldots, \phi\left(c_{n}\right)\right)$. For $T \subseteq \mathcal{T}$, we then define a general invariance condition;

A 7 T-Invariance. For all $N \in \mathscr{N}$, all $i \in N$, all $c, E, \in \mathscr{C}^{N}$ and all $(\phi, \psi, \chi) \in$ $T$ such that $(\phi(c), \psi(E)) \in \mathscr{C}^{N}$,

$$
R_{i}[\phi(c), \psi(E)]=\chi\left[R_{i}(c, E)\right] .
$$

Remark that each invariance conditions we have seen so far can be stated as a $T$-Invariance, where $T$ must of course be adequately chosen. For instance, Scale Invariance is a $T$-Invariance condition with $T=\{(\phi, \psi, \chi) \in \mathcal{T}: \phi=\psi=$ $\chi$ and $\phi(x)=a x\}$.

If the set $T$ is very rich, it is clear that no division rule will satisfy $T$ Invariance. The question then arises: which $T$-Invariances do not lead to an impossibility and which division rules satisfy a form of $T$-Invariance. A partial answer is given in the result below.

Proposition 6 If a division rule satisfies T-Invariance, then, for every $t=$ $\left(\phi_{t}, \psi_{t}, \chi_{t}\right) \in T$, we have $\psi_{t}(x)=a_{t} x+b_{t}$ and $\chi_{t}(x)=a_{t} x+b_{t} / n$.

Proof. By definition of a bankruptcy rule and by $T$-Invariance,

$$
\sum R_{i}\left[\phi_{t}(c), \psi_{t}(E)\right]=\psi_{t}(E)=\sum \chi_{t}\left[R_{i}(c, E)\right] .
$$

Since $E=\sum R_{i}(c, E)$, we can write $\sum \chi_{t}\left[R_{i}(c, E)\right]=\psi_{t}\left[\sum R_{i}(c, E)\right]$. This Pexider equation has as general solution $\psi_{t}(x)=a_{t} x+b_{t}$ and $\chi_{t}(x)=a_{t} x+b_{t} / n$.

This result shows that we have very little choice for $\psi$ and $\chi$ while, for $\phi$, there is no restriction. But, if we want to use a sensible and interpretable, invariance condition, we probably have to choose $\phi$ in the same family of transformations as for $\psi$ and $\chi$, i.e., a postive affine transformation.

\section{Share Invariance}

Another invariance condition is motivated by the following comment of [Young, 1987, p.331].

Equity is relative. People tend to evaluate how fairly they are treated, not in absolute terms, but in relation to how others are treated. 
This suggests that we should reason in terms of ratios but it is not clear which ratios we should use, and how, in our analysis. It can be "share/demand" or "share of agent $1 /$ share of agent 2" or ... For example, some reasonable conditions stated in terms of ratios are

$$
\frac{R_{i}(c, E)}{R_{i}\left(c, E^{\prime}\right)}=\frac{R_{j}(c, E)}{R_{j}\left(c, E^{\prime}\right)}, \forall c, E, E^{\prime}:(c, E),\left(c, E^{\prime}\right) \in \mathscr{C},
$$

or

$$
\frac{R_{i}(c, E)}{c_{i}}=\frac{R_{j}(c, E)}{c_{j}}, \quad \forall(c, E) \in \mathscr{C},
$$

which both lead to the proportional rule. But Young [1987] also writes

But proportional solutions are not the only plausible ones. Especially when the amounts in question are large and the claims very unequal, a good case can be made that it is fairer for the larger claimants to give up relatively more than the smaller ones.

More formally,

$$
c_{i}>c_{j} \Rightarrow \frac{R_{i}(\gamma c, \gamma E) / \gamma c_{i}}{R_{j}(\gamma c, \gamma E) / \gamma c_{j}}<1, \quad \forall(c, E) \in \mathscr{C}, \forall \gamma>0
$$

and this ratio decreases with $\gamma$. And also

$$
c_{i}<c_{j} \Rightarrow \frac{R_{i}(\gamma c, \gamma E) / \gamma c_{i}}{R_{j}(\gamma c, \gamma E) / \gamma c_{j}}>1, \quad \forall(c, E) \in \mathscr{C}, \forall \gamma>0
$$

and this ratio increases with $\gamma$.

Note that (6), (7) and Scale Invariance all contradict this principle (with Scale Invariance, the ratio does not depend on $\gamma$ ). It is therefore interesting to find a formalization of the principle that equity is relative but that does not impose that large amounts are treated like small ones. Here is such a condition.

A 8 Share Invariance. For all $N \in \mathscr{N}$, for all $c, E, E^{\prime}, F, F^{\prime}$ such that $(c, E),\left(c, E^{\prime}\right),(c, F),\left(c, F^{\prime}\right) \in$ $\mathscr{C}^{N}$ and for all $i, j \in N$,

$$
\frac{R_{i}(c, E)}{R_{i}\left(c, E^{\prime}\right)}=\frac{R_{i}(c, F)}{R_{i}\left(c, F^{\prime}\right)} \Longleftrightarrow \frac{R_{j}(c, E)}{R_{j}\left(c, E^{\prime}\right)}=\frac{R_{j}(c, F)}{R_{j}\left(c, F^{\prime}\right)} .
$$

This condition, like all invariance conditions we have seen so far, is difficult to motivate on purely ethical grounds. It is meant to impose some structure or regularity on division rules when ethically appealing conditions are not strong enough to do the job. Nevertheless, Share Invariance is a weak condition (as we will see, it does not characterize a single rule). It is compatible with many ethically appealing conditions like, among others, Young's condition (formalized by (8) and (9)). Hence, if we need to use an invariance condition, Share Invariance is an interesting one. 


\subsection{Characterization}

If we add a very mild continuity condition, it is easy to characterize all rules satisfying Share Invariance.

A 9 Resource Continuity. For all $N \in \mathscr{N}, R$ is continuous in its last argument.

Proposition 7 A division rule $R$ satisfies Share Invariance and Resource Continuity if and only if it is defined, for all $N \in \mathscr{N}$ and all $(c, E) \in \mathscr{C}^{N}$, by

$$
R_{i}(c, E)=c_{i}\left(\frac{z}{c_{j}}\right)^{k_{i, j}(c)}
$$

where $k_{i, j}$ is a real-valued mapping and, for some $j$ in $N, z$ is the solution of

$$
\sum_{i \in N} c_{i}\left(\frac{z}{c_{j}}\right)^{k_{i, j}(c)}=E .
$$

Proof. For $i$ and $j$ fixed, Share Invariance is equivalent to

$$
\frac{R_{i}(c, E)}{R_{i}\left(c, E^{\prime}\right)}=\psi_{c}\left(\frac{R_{j}(c, E)}{R_{j}\left(c, E^{\prime}\right)}\right) .
$$

Applying Share Invariance several times, we find

$$
\begin{aligned}
R_{i}(c, E) & =\psi_{c}\left(\frac{R_{j}(c, E)}{R_{j}\left(c, E^{\prime}\right)}\right) R_{i}\left(c, E^{\prime}\right) \\
& =\psi_{c}\left(\frac{R_{j}(c, E)}{R_{j}\left(c, E^{\prime \prime}\right)}\right) \psi_{c}\left(\frac{R_{j}\left(c, E^{\prime \prime}\right)}{R_{j}\left(c, E^{\prime}\right)}\right) R_{i}\left(c, E^{\prime}\right) \\
& =\psi_{c}\left(\frac{R_{j}(c, E)}{R_{j}\left(c, E^{\prime \prime}\right)} \frac{R_{j}\left(c, E^{\prime \prime}\right)}{R_{j}\left(c, E^{\prime}\right)}\right) R_{i}\left(c, E^{\prime}\right) .
\end{aligned}
$$

Therefore,

$$
\psi_{c}\left(\frac{R_{j}(c, E)}{R_{j}\left(c, E^{\prime \prime}\right)} \frac{R_{j}\left(c, E^{\prime \prime}\right)}{R_{j}\left(c, E^{\prime}\right)}\right)=\psi_{c}\left(\frac{R_{j}(c, E)}{R_{j}\left(c, E^{\prime \prime}\right)}\right) \psi_{c}\left(\frac{R_{j}\left(c, E^{\prime \prime}\right)}{R_{j}\left(c, E^{\prime}\right)}\right) .
$$

Because $R_{i}(c, E)$ is a continuous function of $E$, for all $x, y>0$, there are $E, E^{\prime}$ and $E^{\prime \prime}$ such that $R_{j}(c, E) / R_{j}\left(c, E^{\prime \prime}\right)=x$ and $R_{j}\left(c, E^{\prime \prime}\right) / R_{j}\left(c, E^{\prime}\right)=y$. So, we can rewrite (10) as $\psi_{c}(x y)=\psi_{c}(x) \psi_{c}(y)$ for all $x, y>0$. This is one of Cauchy's functional equations. On any non-degenerated interval $\left[x_{1}, x_{2}\right], \psi_{c}(x)$ is clearly bounded from above and, so, the functional equation has as unique solution $\psi_{c}(x)=x^{k(c)}$, where $k$ is a positive real-valued mapping. If we let $i$ and $j$ vary, then we must replace $k(c)$ by $k_{i, j}(c)$. We now rewrite Share Invariance.

$$
R_{i}(c, E)=\left(\frac{R_{j}(c, E)}{R_{j}\left(c, E^{\prime}\right)}\right)^{k_{i, j}(c)} R_{i}\left(c, E^{\prime}\right)
$$


Letting $E^{\prime}=C$, we have

$$
R_{i}(c, E)=\left(\frac{R_{j}(c, E)}{c_{j}}\right)^{k_{i, j}(c)} c_{i} .
$$

If $k_{i, j}(c) \equiv 1$, then we obtain the proportional rule.

\subsection{A special case}

Suppose that $E=C$ and, so, each agent receives what he claims. All agents are therefore equally satisfied. Suppose now that $E$ decreases by a very small amount. We have to share this decrease among the agents but, because they are all equally satisfied, we may think there is no reason to share this decrease unequally. Formally, this amounts to

A 10 Equal Treatment at Full Satisfaction. $\left.\frac{\partial R_{i}(c, E)}{\partial E}\right|_{E=C}=\left.\frac{\partial R_{j}(c, E)}{\partial E}\right|_{E=C}$, for all $i, j \in N$.

If, on top of Share Invariance, we impose Equal Treatment at Full Satisfaction, we have

$$
\left.\frac{\partial R_{i}(c, E)}{\partial R_{j}(c, E)}\right|_{E=C}=1=\frac{c_{i}}{c_{j}} k_{i, j}(c)
$$

which implies $k_{i, j}(c)=c_{j} / c_{i}$. We obtain therefore the unique division rule

$$
R_{i}(c, E)=c_{i}\left(\frac{z}{c_{j}}\right)^{c_{j} / c_{i}}
$$

where, for some $j$ in $N, z$ is the solution of

$$
\sum_{i \in N} c_{i}\left(\frac{z}{c_{j}}\right)^{c_{j} / c_{i}}=E,
$$

already introduced by Marchant [2004] and called Gain Proportional. So, we have proved

Proposition 8 A division rule $R$ satisfies Share Invariance, Resource Continuity and Equal Treatment at Full Satisfaction if and only if it is the Gain Proportional rule.

Note that it satisfies Scale Invariance.

\section{Conclusion}

This paper has shown that Scale Invariance is often misunderstood: it is a homogeneity condition and has nothing to do with units of measurement. It does 
not mean that Scale Invariance is irrelevant but, instead, that other invariance conditions might also be interesting. We have presented a few of them and analyzed their consequences. We limited ourselves to conditions similar in some sense to Scale Invariance, i.e., conditions identifying an expression that should be invariant when the resource and/or the claims are modified in some specified way. There might be other interesting invariance conditions with a similar structure and there are of course many other invariance conditions with a differ-

ent structure: for example Replication and Division Invariance [Chambers and Thomson, 2002], Composition [Young, 1988], Consistency [Moulin, 1987], etc.

Acknowledgement. I wish to thank Jean-François Laslier, William Thomson and John Weymark for a number of comments about a previous version. I am also indebted to the participants at a seminar at Université de Caen in February 2005 and at the Indian Statistical Institute (New Delhi) in August 2006.

\section{References}

R. Aumann and M. Maschler. Game theoretic analysis of a bankruptcy problem from the talmud. Journal of economic Theory, 36:195-213, 1985.

C. P. Chambers and W. Thomson. Group order preservation and the proportional rule for the adjudication of conflicting claims. Mathematical Social Sciences, 44:235-252, 2002.

E.J. Friedman. Paths and consistency in additive cost sharing. International Journal of Game Theory, 32:501-518, 2004.

T. Marchant. Rationing : dynamic considerations, equivalent sacrifice and links between the two approaches. Technical report, Working Papers of Faculty of Economics and Business Administration, Ghent University, 2004.

H. Moulin. Equal or proportional division of a surplus, and other methods. International Journal of Game Theory, 16:161-186, 1987.

H. Moulin. Priority rules and other asymmetric rationing methods. Econometrica, 68:643-684, 2000.

H. Moulin. Axiomatic cost and surplus sharing. In K.J. Arrow, A.K. Sen, and K. Suzumura, editors, Handbook of Social Choice and Welfare. Elsevier, Amsterdam, 2002.

L. Narens. Theories of Meaningfulness. Lawrence Erlbaum Associates, Mahwah, New Jersey, 2002.

B. O'Neill. A problem of rights arbitration from the talmud. Mathematical Social Sciences, 2:345-371, 1982.

J. E. Roemer. Theories of distributive justice. Harvard University Press, 1996. 
W. Thomson. Axiomatic and game-theoretic analysis of bankruptcy and taxation problems: a survey. Mathematical Social Sciences, 45:249-297, 2003.

H. P. Young. On dividing an amount according to individual claims or liabilities. Mathematics of Operations Research, 12:398-414, 1987.

H. P. Young. Distributive justice in taxation. Journal of Economic Theory, 44: 321-335, 1988. 